\title{
Antiphospholipid syndrome presenting as episodic limb ischaemia
}

\author{
R E Evans, M J Dillon, M Alfaham
}

\begin{abstract}
A child presented with ischaemic episodes of his left leg from the age of 2 months. He was found to have raised anticardiolipin antibodies so was started on low dose aspirin. At a three year follow up he was asymptomatic with a normal anticardiolipin antibody level while taking aspirin daily, probably for life.

(Arch Dis Child 1996;75:342-343)
\end{abstract}

Keywords: antiphospholipid syndrome, acute limb ischaemia.

The antiphospholipid syndrome was first described in 1983 by Hughes et al in patients with systemic lupus erythematosus (SLE). ${ }^{1}$ Subsequently antiphospholipid antibodies have been found in various other conditions and can also occur in the so called primary antiphospholipid syndrome. In adults the commonest presentations of the latter are venous thrombosis, arterial occlusion, and repeated spontaneous abortions. There are few reports in children.

We report a case of primary antiphospholipid syndrome presenting as episodes of acute distal limb ischaemia in an infant.

\section{Case report}

A thriving baby boy presented at the age of 5 months with a three month history of the whole of his left leg (from hip to toes) becoming mottled, then turning blue and cold, followed by a gradual return of colour. These episodes lasted from a few minutes to $30 \mathrm{~min}$ utes and occurred on average twice a week. $\mathrm{He}$ cried in distress when his limb was blue. He had no episodes while asleep and was well between episodes. He was his mother's second livebirth. She had not had any miscarriages; her only problem during pregnancy had been vomiting which had required admission for intravenous fluids at five months' gestation. $\mathrm{He}$ was a term, normal delivery with a birth weight of $3400 \mathrm{~g}$ and had an uncomplicated neonatal period. He was breast fed until the age of 3 months. At the age of 4 weeks he was briefly an inpatient with vomiting which was thought to be due to a viral illness. At 2 months he suffered a minor accidental head injury. There was no family history of note.

Examination revealed a well looking baby boy with weight and head circumference on the 50 th centile and length on the 97th centile. His limbs were not mottled or cyanosed. His femoral pulses and four-limb blood pressure meas- urements were normal. The rest of the examination was entirely normal.

Since these episodes sounded ischaemic rather than being associated with temperature or autonomic immaturity as commonly seen in babies, we undertook further studies. Ultrasonography excluded a pelvic mass, and neither immersion in cold water nor compressing his femoral vessels precipitated similar episodes. Doppler studies of the femoral arteries and veins, echocardiography, and electrocardiography were all normal. His blood count was normal except for a platelet count of $600 \times$ $10^{9} / 1$. The erythrocyte sedimentation rate (ESR) was $3 \mathrm{~mm} /$ hour, there was a normal clotting screen, a negative autoimmune profile, and no anti-DNA antibodies were detected. IgG anticardiolipin antibody was raised at $\mathbf{1 4 . 9}$ AEU (the upper range of normal being 7), with normal levels of IgM anticardiolipin antibody. However, by this time the episodes had stopped so the significance of the raised IgG anticardiolipin was questioned. The test was repeated at 9 months of age when the IgG anticardiolipin had risen to 26.5 AEU. We therefore diagnosed antiphospholipid syndrome without an obvious precipitating cause. Because of the risk of thrombosis he was started on low dose aspirin, $50 \mathrm{mg}$ once daily.

During the first few months on aspirin there were daily episodes of blue hands and feet, lasting up to a minute but not associated with crying. These gradually disappeared by the age of 2 years and there have been no further ischaemic episodes since. His platelet count returned to normal by 17 months and his IgG anticardiolipin was within the normal range by 28 months.

At 3 years and 8 months old, he was symptom-free on aspirin.

\section{Discussion}

Antiphospholipid antibodies are a diverse group, of which anticardiolipin and the lupus anticoagulant were the first recognised, hence the old name of anticardiolipin syndrome for this condition. ${ }^{2}$ The stimulus for the production of these antibodies is not known but there could be a genetic predisposition. There have been reports of antiphospholipid positive families with possible associations with certain HLA types, namely DR7, DR4, DQw7, and DRw53. ${ }^{2}$ The IgM anticardiolipin has been found to rise transiently in viral infections, but no such rise has been shown for the IgG form (the form which is usually raised in the antiphospholipid syndrome).

Dr Alfaham. 
Infants can acquire the IgG form of anticardiolipin transplacentally if the mother has antiphospholipid syndrome, the quantity of the antibody transferred being proportional to the maternal level. ${ }^{3}$ Thromboembolic episodes have been reported in some of these infants, but the majority remain symptom-free. IgG levels start falling at 3 months and are undetectable by 6 months. Our case cannot be attributed to passive acquisition of maternal IgG since the raised level was first detected at 6 months and had risen further by 9 months.

Beyond the neonatal period there have been only a few reports in the paediatric literature, the majority of which are of central nervous system events, for example cerebral ischaemic infarction, spinal cord infarction, and chorea. Other reported cases include Budd-Chiari syndrome $^{4}$ and myocardial infarction in young children. There is only one report of acute distal ischaemia as a presentation of antiphospholipid syndrome in a child. ${ }^{5}$ A review of the paediatric literature on antiphospholipid syndrome $^{4}$ described an equal sex ratio in children, compared with the female preponderance of 2 to 1 in adults, with an incidence of deep venous thrombosis in children of $44 \%$ compared with $54 \%$ in adults.

How these antiphospholipid antibodies produce thrombosis is unknown. The antibodies may have a direct effect on platelets, on endothelial cells, or on the clotting mechanism itself. $^{6}$ However, whatever the mechanism, apolipoprotein- $\mathrm{H}$ has been shown to be an essential cofactor ${ }^{2}$ and antiphospholipid antibodies cross react with oxidised low density lipoproteins, suggesting a possible role in the actual pathogenesis of atheroma. ${ }^{2}$

It is not clear how anticardiolipin antibodies produced the transient distal limb ischaemic episodes seen in this case. Since the longest episode lasted 30 minutes it is difficult to attribute the blame to arterial thrombi. We speculate that the antibodies may make the vessel more prone to spasm. Another possible explanation could be that the antibodies lead to the formation of microthrombi which reduce the blood vessel lumen, so that if the vessel constricts for some other reason it is more likely to lead to occlusion.

As only a proportion of individuals with antiphospholipid antibodies develop thrombosis it is debatable whether they should be treated in the absence of thrombotic episodes. However, we recommend treating those with antibodies with low dose aspirin, and those with recurrent thromboses with anticoagulants such as warfarin. The duration of treatment, especially when the anticardiolipin IgG level becomes normal, is arguable. Since the natural history of these antibodies, in the absence of aspirin, is to persist for years or even for life, the current recommendation is to continue treatment indefinitely.

We thank Miss Hayley James for her help in preparing the manuscript.

1 Hughes GRV. Thrombosis, abortion, cerebral disease and

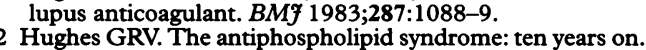
Lancet 1993;342:341-4.

3 Zurget N, Bakimer R, Tincani A, et al. Detection of Zurgil N, Bakimer $R$, Tincani $A$, et al. Detection of
anti-phospholipid and antiDNA antibodies and their anti-phospholipid and antiDNA antibodies and their
idiotypes in newborns of mothers with anti-phospholipid syndrome and SLE. Lupus 1993;2:233-7.

4 Saca LF, Szer IS, Henar E, et al. Budd-Chiari syndrome associated with antiphospholipid antibodies in a child: report of a case and review of the literature. $\mathcal{F}$ Rheumatol 1994;21:545-8.

5 Mechinaud-Lacroix F, Jehan P, Debre MA, et al. Thrombotic manifestations and acute distal ischaemia in primary antiphospholipid syndrome in children. Arch Franc Pediar 1992;49(suppl 1):257-9.

6 Hughes GRV. Antiphospholipid antibody syndrome. Med Int 1994;22:65-6. 